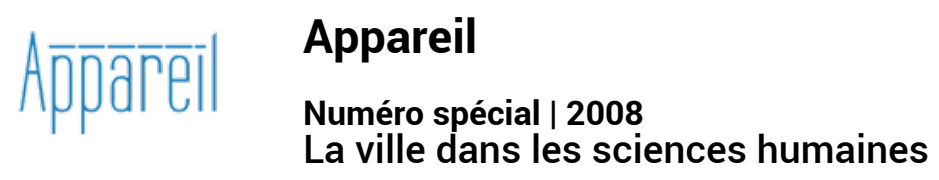

\title{
La ville appareillée : Arendt, $W$. Benjamin et Baudelaire
}

Jean-Louis Déotte

\section{OpenEdition}

1 Journals

Édition électronique

URL : http://journals.openedition.org/appareil/449

DOI : 10.4000/appareil.449

ISSN : 2101-0714

Éditeur

MSH Paris Nord

Référence électronique

Jean-Louis Déotte, «La ville appareillée : Arendt, W. Benjamin et Baudelaire », Appareil [En ligne], Numéro spécial | 2008, mis en ligne le 30 juin 2008, consulté le 30 juillet 2020. URL : http:// journals.openedition.org/appareil/449; DOI : https://doi.org/10.4000/appareil.449

Ce document a été généré automatiquement le 30 juillet 2020.

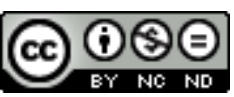

Appareil est mis à disposition selon les termes de la Licence Creative Commons Attribution - Pas d'Utilisation Commerciale - Pas de Modification 4.0 International. 


\title{
La ville appareillée : Arendt, W. Benjamin et Baudelaire
}

\author{
Jean-Louis Déotte
}

\section{Arendt}

1 Comme le montre Georgia Athanasopoulou dans ce même numéro, la Cité grecque telle que la conçoit Arendt dans Condition de l'homme moderne est un mode d'espacement spécifique, ouvert essentiellement à l'accueil de l'action, c'est-à-dire à l'événement politique. Dans la hiérarchie des modes de l'activité humaine, tout se comprend chez Arendt en fonction du primat aristotélicien de l'action sur le poème, la praxis l'emportant sans conteste sur la poïssis. L'artiste est une sorte d'artisan qui, comme tout homo faber, élabore de l'œuvré, c'est-à-dire un artefact qui, à la différence du produit du travail, ne sera pas consommé immédiatement. L'œuvré est destiné à perdurer, c'est le cas de tout objet produit par l'artisan. Dans le cas d'une œuvre d'art, outre cette temporalité spéciale, la temporalité d'une chose qui résiste au temps, l'autre aspect est celui de la mémoire: l'œuvre d'art, le poème épique par exemple, parce qu'elle enchaîne sur de hauts faits politiques et guerriers, transmettra la trace de l'agir inouï aux générations à venir. À la limite, pour Arendt, l'art n'est pas plus politique que ne l'est la législation d'une Cité rendant possible l'action. Si la constitution légale, le nomos, d'une cité grecque détermine les contours de l'action à venir, l'œuvre rappelle les grandes actions du passé qui peuvent servir de modèle. On est dans l'horizon spatial de la Cité (polis) et dans l'ouverture au passé (transmission). Ce sont d'ailleurs les deux axes rendant possibles l'action. On pourrait dire que chez Arendt, la loi et l'art sont du côté de l'appareil urbain pré-politique, tandis que l'agir est du côté du surgissement du nouveau : l'apparition d'un nouveau monde telle que le pose chaque action inattendue, discontinue et ouverte à un enchaînement improbable du fait de la pluralité humaine.

On peut en effet isoler deux propositions essentielles dans le texte d'Arendt Qu'est-ce que la politique?, qui prépare La Condition de l'homme moderne:

1) La politique repose sur un fait: la pluralité humaine. Dieu a créé l'homme, les hommes sont un produit humain, terrestre, le produit de la nature humaine. 
3 Arendt indique ainsi que le politique ne s'intéresse pas à l'essence de l'homme, ce qui au contraire relèvera de la théologie, de la philosophie, des sciences humaines, etc. Bien sûr, la philosophie s'attachant à la politique, d'ailleurs pour en nier la spécificité ou la légitimité (Platon, La République) a toujours été obligée de prendre en considération des modes d'être de la pluralité : le peuple et les différents régimes du politique chez Platon ou Aristote, l'«entre » rendant inévitable la lutte de tous contre tous chez Hobbes (Léviathan), la nécessité du contrat chez Rousseau, qui suppose fatalement l'existence d'un être-ensemble a priori pour que les hommes puissent passer contrat, et donc la nécessité du langage (Le Contrat social), la masse prolétarienne et ses rapports à la conscience de classe chez Marx (Le 18 Brumaire de Louis Napoléon Bonaparte), etc.

Arendt, en pointant la séparation, l'espace entre chacun, désanthropologise radicalement la formule de Kant, quand ce dernier parlait de l'« insociable socialité » de l'homme. Elle insiste sur la capacité de faire du lien sur et à partir de la division et la capacité de la division de délier tous les rapports entre les hommes. Ce qui interdit, selon Arendt, de donner une définition, même politique de l'homme. L'homme n'est pas un « vivant politique » comme l'écrit Aristote. L'homme n'a pas de substance avérée. Mais alors, d'où tient-il sa détermination? D'une manière évidente, Arendt établit l'horizon de l'agir humain: comme ce sera un inter-agir, le monde auquel appartiennent les agissants privilégiera l'espace, la synchronie et non le temps. L'histoire, la diachronie, avec un tel point de départ, sera une dimension seconde, d'ailleurs suspecte. S'en remettre à l'histoire, c'est pour elle faire surgir un sujet collectif agissant dans le dos des hommes, sans raison (Kant) ou avec raison (Hegel). L'histoire, ce n'est pas la dimension originaire de l'entre, mais celle de l'enchaînement, avec le risque d'une dépravation de l'enchaînement d'une action sur une autre, toujours improbable, en causalité nécessaire de type physique.

La politique se situe donc entre les hommes: s'agit-il d'un vide comme entre des atomes? D'où la seconde proposition :

2) La politique traite de la communauté et de la réciprocité d'êtres différents. Les hommes dans un chaos absolu ou bien à partir d'un chaos absolu de différences s'organisent selon des communautés essentielles et déterminées ${ }^{1}$.

Mais, avec un tel point de départ, peut-on penser l'effectivité d'une communauté, sa détermination sociale historique?

7 La philosophie a selon elle deux bonnes raisons de ne jamais trouver le lieu de naissance de la politique. La première est :

Le zoon politikon: comme s'il y avait en l'homme quelque chose de politique qui appartiendrait à son essence. C'est précisément là qu'est la difficulté ; l'homme est a-politique. La politique prend naissance dans l'espace-qui-est-entre-les hommes, donc dans quelque chose de fondamentalement extérieur-à-l'homme. Il n'existe donc pas une substance véritablement politique. La politique prend naissance dans l'espace intermédiaire et elle se constitue comme relation. C'est ce que Hobbes avait compris.

La seconde : si la pluralité n'est pas a priori, mais l'homme tel qu'il est créé par Dieu, alors ce qui importe, c'est le Même. Autrui, c'est le même que moi, une créature tout aussi méprisable et donc à combattre ${ }^{2}$.

8 La politique n'est pas une qualité que possède la substance « homme ". La politique, c'est le Mitsein, c'est l'espacement. C'est pour cette raison que l'irruption des masses et surtout des foules insurrectionnelles et fusionnelles que décrivait Canetti n'est pas en elle-même politique. 
9 Il faut rappeler l'horizon d'Arendt : la radicale nouveauté de l'histoire des années 30-50, c'est la disparition de masse (génocides et bombardements atomiques) par quoi s'inaugure sa réflexion sur la politique. À partir de là, Arendt va caractériser l'agir politique en termes d'apparitions, au sens de donner de soi-même aux autres une apparence véridique. Dès lors, la question de la scène où s'exposent les singularités est essentielle, et donc celle de l'espace. Avant d'être une agora, lieu de la persuasion mutuelle, c'est l'exposition de soi, qui est essentielle. La loi la plus fondamentale dirait: expose-toi!

10 C'est dire qu'il y aura des espaces plus ou moins appropriés à la politique. Ne sont pas politiques des espaces, pourtant communautaires, comme l'église ( $d u$ fait du privilège de la transcendance). D'où le refus chrétien de la politique (Tertullien), le privilège du secret de la conscience. Ou, de l'autre côté l'extrême, le marché médiéval, chrétien ou musulman: car l'activité marchande est dédiée à la reproduction de la vie non à l'action (la vita activa). De même pour H. Arendt: l'usine, l'atelier, le bureau, où s'emploie l'animal laborans.

11 C'est dire qu'un espace commun, public, n'est pas nécessairement un espace politique s'il ne permet pas une relation strictement horizontale d'exposition, puis de persuasion: l'horizon est celui d'une multitude, mais cette multitude doit trouver sa finalité dans la persuasion mutuelle. Ce sera bien la question d'un « nous » qu'il faudra aborder en termes de frontières, de limites, de bords.

12 Qu'est-ce qu'un espace politique? C'est un espace où s'adressant à des pairs, la singularité n'est pas dans un rapport de commandement avec quiconque, comme dans l'esclavage, et dans le cadre de la famille du pater familias, ni soumise à quiconque.

13 Dans cette horizontalité absolue, originaire, l'agissant prend la parole ou combat l'ennemi. Cette parole est une action. Parole performative. Cette parole-action ouvre un monde nouveau, à chaque fois.

14 L'action politique interrompt ainsi le devenir physique des choses, la continuité prévisible, et laisse advenir ce qui n'avait jamais été dit ou vu: une nouvelle proposition d'action par exemple. L'action est une véritable apparition.

15 L'exemple princeps d'Arendt de l'espacement entre les singularités est pris dans le monde d'Homère : c'est le principe d'isonomia comme égalité de la parole et non égalité de tous devant la loi.

16 Or, depuis les travaux de M. Detienne ${ }^{3}$, on sait mieux quelle était la configuration de ce «nous» homérique: un cercle centré. Une forme géométrique. Le nous politique est topologiquement appareillé. Les guerriers aristocratiques, archaïques, grecs, se mettaient en cercle au moment du partage du butin ou de la prise de décision. Celui qui allait s'adresser au groupe, au nom du groupe, pour proposer telle ou telle action, se plaçait au centre: ès méson. Le sceptre tenu était le symbole d'une légitimité, celle accordée par le groupe, le temps, limité, de la prise de parole. Arendt donne un second exemple d'espacement politique idéal : elle développe cette figure quand elle aborde la question de la véracité "politique», en fait ontologique : qu'est-ce qui nous prouve, demande-t-elle, que nous appartenons au même monde, à un monde commun? Que la chose saisie par une multitude de regards existe et est la même pour tous ? C'est, écritelle, la certitude, que premièrement, regardant tous la même chose au milieu, en même temps, à partir de places différentes, donc de "perspectives» différentes, nous appartenons au même monde du fait de la convergence des regards. Secondement, c'est 
la même opération, réversible : car regarder de multiples points de vue la même chose, certifie l'essence complexe de cette chose et, à rebours, reconfirme notre appartenance au même monde

Sans s'en rendre compte Arendt reprend pour elle, comme s'il allait de soi, un mode de la mise en espace qui ne surgira en fait qu'à la Renaissance italienne, au $\mathrm{Xv}^{\mathrm{e}}$ siècle, dans des Cités qui réinventeront la politique. Ce mode de la mise en espace, c'est l'appareil perspectif, que les Anciens ne connaissaient pas. Il s'agit en effet de l'articulation spatiale d'une multitude de points de vue qui ne sont comparables que du fait de leur appartenance à un même site, défini géométriquement par le géométral. Ce site n'est pas une articulation de lieux hétérogènes comme dans la pensée antique. L'appareil perspectif, c'est une certaine articulation d'un certain nombre de points (de vue) singuliers et d'un universel. Les premiers artistes à déployer cet espace privilégieront la représentation d'objets géométriques symétriques (temple à "plan centré », villes idéales, etc. ${ }^{4}$ ). L'intérêt d'une telle représentation picturale, c'est que connaissant un point de vue sur l'objet, en en ayant donc une représentation, on peut en déduire tous les autres possibles (de derrière, d'en haut, d'en bas, etc.). L'appareil perspectif permet la comparaison rationnelle de tous les points de vue sur le même objet, parce qu'il génère un site universel.

Pourtant, est-ce qu'une communauté en général ne suppose pas quelque chose comme un sol commun? Oui : mais ce sol n'est pas nécessairement un topos, encore moins un site universel, un géométral. Benjamin affirmera dans ses textes « esthétiques ${ }^{5}$ » de jeunesse qu'un des rapports possibles des hommes à la loi "positive», c'est la circonscription archaïque d'un espace «sacré » se différenciant d'un espace "profane». Ce qui faisait alors le commun de la communauté, ce n'était pas la convergence des regards sur le même, mais le fait d'être passible des mêmes récits, ceux, provenant selon la tradition, des "grands ancêtres » mythiques. L'appareil de la narration ${ }^{6}$, indissociable d'une écriture de la loi sur des supports rendus équivalents (corps, Terre, objets, « fétiches »), rend compte à la fois de l'hétéronomie absolue de la loi (attribuée aux grands ancêtres mythiques), de la passibilité non-démocratique des singularités qui ne peuvent rien y changer, de la hiérarchie des lieux et d'une temporalité spécifique qui est celle du in illo tempore.

On veut montrer par là que si la politique est "à l'extérieur de l'homme ", si « elle prend naissance dans l'espace-qui-est-entre-les hommes", alors cet espace n'est pas quelconque, mais doit être à chaque fois spécifié pour distinguer des époques du politique qui supposeront des époques de l'espacement et donc de la ville. Ce n'est pas un espace immédiatement vécu par tous et par chacun comme le champ perceptif des phénoménologues. L'entre, le mit de Mitsein, ce qui divise tout en liant, n'est pas l'« espace » en général, mais au minimum un certain espace appareillé : le cercle des guerriers aristocratiques grecs, l'espace de représentation des cités italiennes qui réinventèrent la politique, celui des conventions représentatives et des places révolutionnaires françaises, etc. Il y aura autant d'espaces politiques, de figures du "nous », et donc de villes, qu'il y aura d'espaces d'exposition des singularités les unes aux autres.

On voit comment Arendt, trop phénoménologue, fait l'impasse sur les conditions techniques, c'est-à-dire appareillées, de l'exposition, car un appareil a toujours une infrastructure technique, ne serait-ce que géométrique. Et finalement, il n'y a pas de ville et $a$ fortiori de politique sans support d'inscription de l'événement et de la loi. C'est 
ce que l'équipe réunie autour de M.Detienne nous apprend d'Athènes (Les savoirs de l'écriture en Grèce ancienne): la polis, c'est un ensemble d'édifices dédiés à la prise de parole, mais aussi ces grands panneaux de pierre sur lesquels étaient enregistrés les textes de loi et qui étaient destinés aux citoyens alphabétisés.

\section{Benjamin et Baudelaire}

21 C'est Benjamin qui aura le mieux compris que la ville n'existe pas en soi (pas plus qu'une autre chose, phénomène ou apparition), parce qu'elle est toujours appareillée. D'une part au sens où il $\mathrm{y}$ a des époques de la ville qui sont celles de l'appareil historiquement dominant, d'autre part au sens où il $\mathrm{y}$ a une conception-production de la ville à partir de ces appareils.

Les textes de Benjamin sur Baudelaire et Paris, le grand livre inachevé Paris, capitale du $\mathrm{XIX}$ siècle, sont à ce double titre exemplaires.

1) La seconde partie du Paris du Second Empire chez Baudelaire (1938) est consacrée aux thèmes du flâneur et de la trace, thèmes centraux pour l'ouvrage. La première phrase : "Quand l'écrivain s'était rendu au marché, il regardait autour de lui comme dans un panorama " confirme que la perception visuelle de l'écrivain est appareillée par un certain dispositif de spectacle apparu au tout début du xix ${ }^{e}$ siècle à Paris comme à Londres (une invention de Barker, réalisée par les Thayer) : le panorama. Dès lors sa production donnera lieu à une sorte de genre littéraire, les physiologies, une «littérature panoramique ", dont le plus grand représentant est Balzac. Le rapport entre l'appareil architectural et le genre littéraire est structurel :

Ces livres sont faits d'une série d'esquisses dont le revêtement anecdotique correspond aux figures plastiques situées au premier plan des panoramas, tandis que la richesse de leur information joue pour ainsi dire le rôle de la vaste perspective qui se déploie à l'arrière-plan. (p. 55)

Cette littérature succombera vite du fait de son manque de hardiesse, en cette situation politique de "terreur", et de sa naïveté qui consiste à universaliser socialement à partir d'un seul exemple de personnage (Balzac) et laissera la place à une littérature où dominera le roman policier et l'asocial, comme chez Poe, lequel profite de la foule pour ne pas laisser de traces.

Ce qui m'intéresse ici, c'est que le panorama comme architecture est indissociable du passage urbain qui se développera à Paris dans le premier tiers du siècle. Mettons en exergue le Passage des panoramas qui s'ouvre toujours sur les Grands Boulevards parisiens et ses deux rotondes qui, elles, n'existent plus (Germain Bapst, Le guide des passages couverts de Paris, éd. du Mécène, p. 197). Or les passages sont une ville dans la ville, une ville beaucoup plus hospitalière que les rues mal éclairées et souvent dépourvues de trottoirs de l'extérieur. C'est un réseau qui fascine beaucoup Benjamin à l'égal des autres réseaux existants (les carrières souterraines, les égouts) ou à venir, celui des vastes perspectives ouvertes par Haussmann sous le Second Empire, par exemple. Un réseau appareillé en remplacera donc un autre.

Pour l'heure la structure du panorama est identique à celle du passage, à ceci près que le panorama apporte une dimension de réflexivité que ne possède pas le passage, malgré ses alignements de miroirs. Le panorama, lui, est une monade leibnizienne. Un journaliste de l'époque écrivait ceci : 
Le spectateur pénètre dans l'édifice en passant sous la section inférieure du cylindre et n'accède à l'escalier de la plate-forme, qu'en traversant des corridors obscurs et nus, où son œil perd momentanément la mémoire des choses du dehors, où il s'isole, où il oublie la lumière. De la sorte, en arrivant sur la plate-forme, le spectateur est, si l'on peut parler ainsi, envahi par le spectacle offert à ses regards ; son œil s'en repaît avidement, il l'y promène avec le plaisir qui accompagne l'accomplissement d'une fonction dont l'exercice nous a été passagèrement interdit ; et aucun objet juxtaposé ne venant lui rappeler qu'il est en présence d'une peinture fallacieuse, il s'abandonne à l'illusion que l'artiste a cherché à éveiller en lui, et qui l'étreint, d'autant plus puissante et durable que l'exécution de la peinture elle-même est plus parfaite et plus savante. ville, mais en penseur des grandes formes de l'expérience et de la transmission de l'expérience, qu'il distingue fortement de la question de la communication de l'information (les média comme la grande presse). C'est dire que pour lui le panorama relève d'une philosophie de la vérité :

L'intérêt pour le panorama vient de ce qu'on voit la vraie ville, la ville à la maison. Ce qui se trouve dans une maison sans fenêtres est le vrai. Du reste, le passage aussi est une maison sans fenêtres. Les fenêtres à l'étage qui donnent sur le passage sont comme des loges d'où l'on peut voir à l'intérieur, mais non à l'extérieur de celui-ci. (Le vrai n'a pas de fenêtres ; le vrai ne donne nulle part sur l'univers). (Paris, Q 2a, 7, p. 548)

Dès lors, comme le montrera la seconde étude sur Baudelaire, Sur quelques thèmes baudelairiens (1939), si le flâneur est le véritable héros baudelairien, cette figure n'est identifiable que sur un fond de perception, ce que Benjamin nomme un voile, constitué par la masse (ou la foule). Or la foule est le nouveau mode d'être de la multitude. Ou pour le dire en termes arendtiens, c'est un certain registre de l'espacement. Registre caractérisé par sa compacité, par un certain mouvement, voire par sa vitesse, par l'indifférence aux autres mêlée d'une identification sociale immédiate. Nul n'y est un strict étranger aux yeux des autres, même si tous sont des anonymes. La foule est donc encore un certain mode de l'apparaître réciproque du fait de son articulation. Telle que la décrit Benjamin, c'est un voile qui à la fois cache la réalité de la ville, sa brutalité, engloutissant en son sein le criminel ou l'asocial, mais qui en même temps pousse en avant telle ou telle singularité, la faisant comparaître. Ce qu'évoquera puissamment le sonnet À une passante de Baudelaire. La foule est donc une certaine détermination du milieu de perception, auquel tous participent, puisque le poète est aussi un homme de la foule. Pour Baudelaire, elle est aussi « naturelle » que le seront la mer, la forêt ou le monde des esprits pour Hugo. La consistance de ce milieu de perception est chez Benjamin quasi ontologique (cf. notre : Esthétiques benjaminiennes, 1997). Or, malgré les apparences, la foule ne constitue pas un véritable réseau dont les éléments seraient en voie d'individuation. Pour le dire en termes empruntés à Simondon, ce n'est pas un milieu transindividuel comme le serait une véritable communauté en voie d'individuation. La foule ne se constituant en groupe qu'à l'occasion d'un incident: alors, momentanément, du fait d'un événement de pur hasard comme un accident de la 
circulation, les individus se regroupent avant de passer leur chemin. Pour Benjamin, cet agrégat, qui n'a pas en lui la raison de s'assembler, sera la proie des partis totalitaires $\mathrm{du} \mathrm{xx}^{\mathrm{e}}$ siècle qui lui donneront une identité artificielle : la race, un peuple fantasmé.

2) Pour rendre compte de ce mode de l'être-ensemble, Benjamin en se référant aux études de K.Kraus sur la grande presse (laquelle livre des informations brutes de décoffrage, des événements "en soi", non intégrés dans une narration, non reliés puisque la première page d'un journal est un pur spectacle de l'hétérogène, dans un jargon journalistique quasi inintelligible, etc.) montre que les événements ne peuvent plus donner lieu à une expérience réelle du psychosocial. Dès lors, l'expérience qu'un individu peut avoir du monde se limite à la sphère privée, laquelle est dissociée de la sphère collective. On assiste à une dissociation de l'expérience privée et de l'expérience collective. Bien davantage, puisque l'expérience d'un individu ne peut plus s'intégrer dans l'expérience collective, puisqu'on ne peut plus narrer les événements, la psychologie comme discipline du savoir s'émancipe de l'étude du social, laquelle est condamnée alors depuis sa fondation durkheimienne à se demander comment restaurer le lien social.

La poésie de Baudelaire correspondrait à ce moment du déphasage du psychosocial en psychologie d'un côté, sociologie de l'autre. On peut faire l'hypothèse que Benjamin cherche ici à restaurer un état du psychosocial plus essentiel.

3) Si la dimension sociale, en fait communautaire, n'est plus constitutive de l'expérience d'une singularité, cette dernière est totalement appauvrie. La psyché en est réduite à subir les événements dans leur aspect le plus destructeur: le choc émotionnel. L'expérience urbaine la plus générale est celle de l'entrechoc du fait de la réception des média de communication et du contact de la foule. La conscience comme système psychique cesse d'être une faculté dédiée à la connaissance. Ainsi n'est-elle plus caractérisée par la psychanalyse selon la dimension d'intentionnalité, de visée, que décrit la phénoménologie husserlienne, la conscience ne caractérise plus notre rapport au monde et aux autres, mais est décrite par Freud que Benjamin mobilise, en particulier à partir de Par delà le principe de plaisir, comme un dispositif de pareexcitations dont la fonction est de préserver les équilibres psychiques internes. Une image approximative consisterait à dire que chacun s'enferme dans une bulle protectrice (métaphore que filera Sloterdijk). Plus sérieusement, mobilisant Proust et sa forte distinction entre mémoire volontaire et mémoire involontaire, Benjamin affirmera que la connaissance empirique, diurne, celle que caractérise la mémoire volontaire des événements réduits à du vécu, cette connaissance n'a que la consistance de la cendre d'un souvenir sans vie : un souvenir qui n'a pas la productivité poétique d'une trace. La thèse est abrupte: l'habitant des villes modernes sait qu'il ne peut compter sur sa mémoire des traces et qu'on ne conservera aucune trace de son existence puisqu'en l'absence de narration, c'est-à-dire en l'absence de l'appareil communautaire rendant possible l'expérience au sens de la tradition, il n'y a plus de surface d'inscription des traces. Ne subsiste pour lui que l'illusion de laisser des souvenirs par le biais de la possession de ses chers objets, qu'il va chercher à préserver en leur donnant à tous des étuis, jusqu'à transformer son propre appartement en un étui comme une coquille pour une existence appauvrie, jusqu'à devenir lui-même un «homme-étui ». Or, le flâneur habite le passage urbain comme le bourgeois son appartement: l'un comme l'autre sont abandonnés à une rêverie collective que 
Benjamin n'appelle pas idéologie, mais fantasmagorie parce qu'elle est le produit d'un appareil de projection nouveau: le passage-panorama. Rêverie indissociable d'une nouvelle époque de la ville qu'on aurait tort de réduire à la généralisation de la marchandise et de l'industrie.

4) On peut tirer deux faits majeurs du rappel de ces analyses de la ville.

- Cette philosophie de la ville s'établit à partir de ce qui n'est pas elle : le monde des paysans, des artisans et des marins, monde de la transmission réelle de la profondeur des événements puisque ceux-ci proviennent des lointains géographiques ou du passé. Monde de l'artisanat, de la valeur de culte, où les choses ont le temps de s'accomplir par rapport au caractère de nouveauté de la marchandise.

5 - Dès lors, Benjamin du haut d'une conception du psychosocial dont l'armature traditionnelle est constituée par l'importance de la transmission par narration comme il l'a montré dans Le Narrateur, développe au moins dans un premier temps une critique des dispositifs de communication de l'information. Ainsi, la littérature (Proust, Valéry) et la poésie (Baudelaire, Hugo) seront au défi de cette dissociation du psychosocial. L'appauvrissement de l'expérience réduite à la sphère privée de la volonté rend nécessaire le développement d'une nouvelle science : la psychanalyse. C'est la raison pour laquelle le second texte sur la ville (Sur quelques thèmes baudelairiens) donne une telle importance à l'appareil psychique tel que Freud a pu le théoriser depuis L'interprétation des rêves (1900), en dissociant radicalement système conscient d'un côté et enregistrement des traces mnésiques de l'autre. Dissociation que reprendra comme on l'a vu Proust en opposant deux modes de la mémoire : mémoire volontaire/mémoire involontaire. L'écriture des huit tomes de À la Recherche sera comme une ultime tentative pour sauver la narration. Dès lors la théorie freudienne de l'appareil psychique devient pour Benjamin le moyen le plus légitime pour analyser la ville, le terme d'appareil ayant alors le double sens de modèle théorique et d'instance de production esthétique puisque le Surréalisme en sera un rejeton.

On peut ajouter que c'est parce que Benjamin tenait le Surréalisme pour «Le dernier instantané de l'intelligentsia européenne » (๕uvres complètes, T2,133) et qu'il présentait Le paysan de Paris d'Aragon comme la meilleure propédeutique à l'étude des passages parisiens, qu'il a laissé toutes les pièces nécessaires pour circonscrire l'appareil qui a généré l'urbanité moderne. Cet appareil s'oppose en tous points à la perspective à point de fuite unique, invention architecturale s'il en est (Brunelleschi, Alberti), laquelle rend possible la représentation de l'espace par la coordination d'un site universel et d'une multiplicité de points de vue égaux en droit (le géométral). Mais si la perspective appareilla la ville depuis le $\mathrm{xv}^{\mathrm{e}}$ siècle, ce fut davantage comme projet (les tableautins "Villes idéales ») que comme effectivité. Peut-être d'ailleurs que le $\mathrm{xIx}^{\mathrm{e}}$ siècle sera paradoxalement le grand siècle des perspectives urbaines, mais comme idéalités réalisées avec beaucoup de retard. Ces perspectives urbaines seront en fait elles aussi des rêves urbains comme le laisse entendre Benjamin dans Paris à propos d'Haussmann, comme si l'appareil du passage avait absorbé l'appareil de la perspective. Or le passage défait la conjonction entre représentation d'un espace homogène (car géométrique, isotopique et infini : anti-aristotélicien) et la multiplicité des points de vue. L'appareil du passage désobjectivise les choses puisqu'il transforme pour la masse et du fait de la masse les produits du travail qui y sont exposés en fétiches et qu'il élargit à l'ensemble de la ville la prostitution féminine. Bref, si l'appareil perspectif avait pu servir d'infrastructure à la science moderne (Galilée-Descartes), l'appareil passage générant 
une prodigieuse rêverie collective, replacera l'onirique et ses métamorphoses (Granville) au devant de la scène. Pour Benjamin, penseur de l'histoire, le passage parisien aura été le lieu où le XIX ${ }^{e}$ siècle aura rêvé et enfanté le $\mathrm{xx}^{\mathrm{e}}$ siècle : proposition dont on n'a pas fini d'analyser toute la profondeur. Monde de l'immanence absolue, comme celui que décrira Lyotard dans L'économie libidinale (1974). Un monde de l'intérieur sans articulation du symbolique.

37 5) Le monde des passages renie le schème universel du géométral en transformant toutes les réalités en fantasmagorie. Si le psychosocial perdure, c'est sur le mode de l'onirique.

38 En effet, c'est la masse concentrée dans les passages qui génère le fétiche, c'est-à-dire «l'âme de la marchandise » (Marx) : le fétiche étant distingué de la marchandise, en l'amenant à se prostituer, comme elle le fait avec la femme placée sous les désirs masculins, laquelle devient un objet sexuel en se chargeant d'aura sexuelle. En retour, marchandise et prostituée entraînent l'homme dans une fantasmagorie, comme le ferait une drogue. Si l'on suit à la lettre Benjamin, ce serait dire que le « fétichisme » de la marchandise et la prostitution généralisée sont produits par la masse en circulation dans ses boyaux sans horizon que sont les passages, du fait de la masse d'affects projetée et convergeant sur la marchandise ou la femme, lesquelles, sinon, en ellesmêmes, ne seraient pas fétiches mais simple valeur d'usage ou existence humaine. Le mystère du fétichisme ne résiderait pas dans l'oubli des rapports de production (Marx), mais dans la ville qui est appareillée d'une certaine manière à partir du moment où les rues s'invaginent, où les façades des immeubles disparaissent, où la lumière cesse d'être solaire pour devenir artificielle (éclairage au gaz), où la déambulation se fait extrêmement lente. Ce qui est commun à ces deux éléments majeurs de la fantasmagorie, c'est la projection d'un désir collectif sur une chose qui, de ce fait, concentre une énergie sans pareille et la diffuse en retour vers la multitude qui se trouve ainsi droguée (fantasmagorie). Que l'on soit encore dans l'ère de la projection et de la réflexion miroirique, cela est annoncé dès le début du texte dans la comparaison entre Baudelaire et Blanqui : Benjamin parle alors de « la mise au point de l'image de Baudelaire ». L'image devenue nette (p. 31) est celle de Baudelaire comme conspirateur. Baudelaire pouvant reconnaître ses futurs lecteurs, mais restant inconnu d'eux comme Blanqui voyant défiler sur les Champs Élysées son armée secrète qui ignorait l'identité de son chef.

Cette ville ne peut plus être le lieu d'une connaissance exacte puisque domine la rêverie collective. Benjamin ne dit-il pas d'une chose qu'elle a de l'aura quand on lui fait lever les yeux du fait qu'on la regarde? Ce faisant, ajoute-t-il, celui qui se projette ainsi sur elle se trouve entraîné vers les lointains fantasmagoriques. En rapport avec le nouveau statut de la trace, un nouveau mode de l'aura surgit: une aura qui n'est plus l'apparition proche d'un lointain, mais une aura fantasmagorique (Ilaria Brocchini, Trace et disparition à partir de l'œuvre de Benjamin, 2006).

A contrario, un appareil comme la photographie qui brise l'échange des regards puisqu'il ne répond pas au regard de celui qui est photographié, cet appareil n'est-il pas en son fond, émancipateur : ne générant que des archives objectives, non auratiques ${ }^{7}$ ?

41 Conclusion : Benjamin a produit en creux le modèle de l'appareil "passage" en utilisant la problématique freudienne de l'appareil psychique. Ce faisant, il donne une autre assiette à la vérité : non plus la rationalisation et la certitude de l'ego cogito cartésien qui s'opposaient aux savoirs incapables de donner raison d'eux-mêmes (les illusions des 
sens, le pouvoir des histoires, etc.), mais l'écriture proustienne qui perlabore un savoir à partir des traces mnésiques inconscientes. Le passage est un véritable appareil configurant à nouveaux frais l'événement et non un simple dispositif urbain parce que modifiant le milieu de la perception, il a rendu possible le renouvellement d'un genre littéraire : la poésie lyrique.

Au minimum, comme le rappelle Daniel Payot (Des villes refuges, 1992), une ville c'est donc un certain mode d'espacement et un certain régime de la surface d'inscription. De ce point de vue, la description arendtienne de la polis grecque reste trop encadré par un rapport au symbolique fort traditionnel.

6) Mais chez Benjamin, les notions se dédoublent toujours en modes opposés (deux modes de la trace, de l'aura, de la manifestation des œuvres, du présent, de l'activité pratique, etc.). J'ai montré dans Le Musée, l'origine de l'esthétique (1993) que Benjamin caractérise l'ensemble passage-panorama comme la préhistoire de la modernité, c'està-dire comme une véritable matrice productrice inconsciente d'images caractérisées par leur immanence. Ce milieu de l'imaginaire, comme l'indique le terme de fantasmagorie, ne cesse pas d'être projectif, par un rabattement de tous les comportements urbains selon un principe d'inclusion, par une absence d'extériorité.

En même temps, opposée à la fantasmagorie, une nouvelle sensibilité commune a fait époque et sur le plan de la connaissance, un nouveau principe d'appariement a vu le jour, c'est-à-dire un nouveau mode de réduction de l'hétérogénéité du monde selon un principe de similarité qui n'est plus hiérarchique. C'est la raison pour laquelle l'écriture benjaminienne place tous ses matériaux sur un plan d'égalité, de là l'utilisation de citations sans guillemets. L'acception de la beauté devient tout autre: une beauté ordinaire et banale. Essayons de comprendre les enjeux anthropologiques et politiques de l'œuvre inachevée de Benjamin : le Livre des passages.

On l'a dit, les passages urbains sont des entités architecturales privatives de toute destination cultuelle puisque dans ces rues invaginées, les façades des monuments disparaissent, or c'est par sa façade qu'un monument expose sa destination, sa fonction de culte. Certes, les passages ont une fonction de communication et d'exposition (la prostitution générale), mais il faut désintriquer les deux logiques. C'est dire que l'exposition des marchandises a un revers inaperçu : la circulation adestinale du flâneur qui n'est pas un consommateur intéressé.

Paradoxalement, mais c'est un paradoxe qui donne la clef du poème Manuel pour les habitants des villes de Brecht cité à la fin Expérience et pauvreté (1933) où Benjamin rappelle comme une complainte le mot d'ordre brechtien "Efface tes traces!", le flâneur ne déroule pas ses pensées dont l'accumulation donnerait lieu à de nouveaux paragraphes dans une méditation écrite. Au contraire, le flâneur idéal doit désapprendre toute sa connaissance de Paris (Chroniques berlinoises, in: Écrits autobiographiques, 1990, p. 249-250)! L'enjeu n'est plus la connaissance de soi ou du monde, mais le surgissement d'un sujet esthétique qui est aussi politique. Le flâneur doit suspendre sa capacité cognitive, et donc tout recours à la mémoire volontaire, s'il veut pouvoir être affecté par ce qui apparaît comme phénomène et comme événement. La ville cesse d'être un classique théâtre de mémoire (F.Yates, L'art de la mémoire, 1966).

Cela suppose déjà une révolution de la surface d'inscription. Si Benjamin peut se revendiquer de Loos qui avait déclaré que "l'ornement est un crime", c'est que la revendication d'une architecture méditerranéenne est devenue évidente depuis le séjour de Benjamin à Ibiza ${ }^{8}$, dès lors les matériaux des façades urbaines (le verre) et 
d'une manière générale la surface d'inscription sont libérés de toutes traces et icônes destinales. Dans ces conditions, le flâneur devient le paradigme de l'homme de la démocratie, celui pour lequel le centre est partout et nulle part, et ou, pour le dire plus radicalement, le lieu du sens est indécidable.

La définition du centre du pouvoir démocratique comme lieu vide, c'est-à-dire essentiellement in-occupable, a été élaborée par Cl.Lefort à partir de sa grande thèse sur Machiavel (Le travail de l'œuvre Machiavel, 1972). Cette définition prend tout son sens en opposition à la nature du pouvoir dans les sociétés théologico-politiques chrétiennes où le lieu du pouvoir est le corps du prince, lui-même vicaire du Christ. Dans ce type de régime, la nation s'incarnait dans le corps immortel du roi ; le pouvoir, la loi, le savoir, étant essentiellement intriqués. Dans ce monde où tous les édifices sont alors de destination comme dans Paris décrit par l'historien John W.Baldwin ${ }^{9}$, le type du flâneur ne saurait émerger. Faut-il en plus mobiliser la description de Notre Dame de Paris comme livre de pierre par V.Hugo ? Une ville pré-moderne est un puissant appareil de destination, répondant aux règles d'une cosmétique, c'est-à-dire d'une onto-théoesthétique parfaitement fixée. Il faudra que cette puissance soit suspendue, et cela commencera au xvIII ${ }^{e}$ siècle avec l'invention du patrimoine et du musée, pour que puisse surgir le comportement de cet homme nouveau dont l'existence ne dépend plus de celle des édifices du culte. Le flâneur urbain n'est rien d'autre que le sujet esthétique décrit par Kant dans la Faculté de juger : celui qui, à l'occasion d'une chose dont il ne saura rien, et parce qu'il n'en saura rien, éprouve un sentiment de plaisir paradoxal : à la fois subjectif et pourtant universalisable sans concept. C'est ce sentiment qui lui fait dire: "c'est beau!" sans que ce jugement soit applicable à la chose qui en fut l'occasion. Le flâneur est donc passible d'occasions: ce n'est pas un amateur recherchant ce qui va provoquer son plaisir, c'est un inventeur de situations inédites où les relations symboliques traditionnelles sont mises à mal.

On pourrait de nouveau proposer un dédoublement : le flâneur est soit le témoin vaincu de 1848 décrit par Dolf Oehler ${ }^{10}$, soit celui qui expérimente positivement les nouvelles conditions esthético-politiques. En premier lieu, tout se présente sous le masque de la nouveauté de la marchandise, mais ce mode de temporalité ne doit pas faire illusion: des réalités ruinées peuvent revenir sous cet aspect. Plus essentielle est l'expérience de l'indétermination des repères symboliques, de l'indétermination croissante dans un espace où les anciens dieux, les vieilles idoles se retirent lentement. D'où la remise en cause du partage social traditionnel entre les classes ou entre les sexes et de celui encore plus anthropologique entre morts et vivants. Ce peut-être la raison pour laquelle le thème de la foule indifférenciée comme de la lesbienne est si important chez Baudelaire ou celui du monde des esprits chez Hugo.

Mais la ville moderne de Benjamin a une histoire, et dans le cas de Paris (ou Lyon) au $\mathrm{XIX}^{\mathrm{e}}$ siècle, une histoire ponctuée d'insurrections populaires écrasées. Certes ce flâneur est devenu une surface d'inscription vierge de tout programme préétabli, il est une sorte de membrane sensible aux affects, et déjà au plaisir du beau. Cette potentialité indéfinie le rend susceptible d'autres affects: à tel coin de rue, il peut avoir le sentiment du déjà $v^{11}$, mais il peut être aussi brutalement envahi par une angoisse sans raison apparente. Là une tragédie a eu lieu, une barricade a été écrasée par la force. Il n'y en a pas d'archives, l'histoire est démunie. Le flâneur se doit de devenir enquêteur, c'est-à-dire historien. L'homme sans destin doit écrire l'histoire à partir de 
traces ininscrites pour que le tort subi par les vaincus de l'histoire ne reste pas sans témoins.

51 8) Pour résumer, où pourrait dire que la ville, parce qu'elle est un certain mode d'espacement (et la négation de cet espacement fait encore ville comme dans le cas de la ville théologico-politique) a vu surgir des appareils urbains comme la perspective ou le passage. Ces appareils strictement urbains ont été interprétés par d'autres appareils qui ne l'étaient pas : la peinture perspectiviste dans l'Europe du sud, la camera obscura dans l'Europe des Flandres et des Pays Bas, le musée, le panorama, la photographie, la psychanalyse, le cinéma, etc. Cette seconde série d'appareils apporte une dimension réflexive que les appareils strictement urbains n'auraient pas en eux-mêmes. Dès lors la définition leibnizienne de la Ville est parfaitement recevable :

Et, comme une même ville regardée de différents côtés paraît tout autre, et est comme multipliée perspectivement; il arrive de même, que par la multitude infinie des substances simples, il y a comme autant de différents univers, qui ne sont pourtant que les perspectives d'un seul selon les différents points de vue de chaque Monade. (\$57 Monadologie)

On en conclura qu'il y a autant de villes que d'appareils et de régimes de la surface d'inscription pour les exprimer et les produire. On ajoutera à Leibniz que nos villes historiques se résument à des strates différemment appareillées, non pas suivant une compréhension archéologique comme tente de le faire encore Freud (Malaise dans la civilisation) quand, à propos de Rome, il imagine qu'en un même lieu du Forum on puisse restituer des édifices d'époques totalement différentes. Cette impossibilité matérielle étant là pour rappeler que les traces mnésiques peuvent se superposer dans une nouvelle tentative de nous donner des représentations de l'appareil psychique. C'est maintenant la ville qui permet d'approcher l'appareil psychique.

Dès lors, on pourrait faire l'hypothèse que si une ville c'est un certain espacement ouvert à l'événement, alors ce entre quoi il y a des écarts, c'est entre des productions historiques d'appareils d'époques différentes. Une ville, c'est déjà le partage entre un espace profane et un espace sacré du fait de l'appareil narratif, ce sont aussi, du fait d'un rapport moderne aux ruines (Riegl), rapport qui est en son fond muséal, des valeurs différentes de conserver le patrimoine et il y a bien des villes musées comme Venise ou nos centres historiques. D'autres textes de Benjamin ouvrent d'intéressantes pistes de réflexion. Dans Petite histoire de la photographie, les places et rues parisiennes désertes, photographiées par Atget, deviennent autant de preuves que là un crime a été commis et que le cadavre a disparu. Comme si après les massacres de la Semaine sanglante de 1871 et l'ouverture d'immenses fosses communes, alors donc qu'il n'y a plus de traces des vaincus de l'histoire, plane au-dessus des lieux urbains un affect d'angoisse provoqué par la disparition de masse.

Et dans L'œuvre d'art à l'époque de sa reproductibilité technique, texte essentiel sur le cinéma, Benjamin écrit que le cinéma et l'architecture ont en commun de rendre impossible une perception volontaire et attentive et de libérer une perception de distraction, non focalisante et tactile, ce qui nous ramène vers la mémoire involontaire de Proust et ses hasards. On peut postuler que le flâneur et l'homme ordinaire au cinéma ne font qu'un. Car l'homme ordinaire du cinéma ${ }^{12}$, le spectateur anonyme, a tous les traits d'un flâneur immobile découvrant toutes les beautés démocratiques de la ville moderne ${ }^{13}$. 
ors, on pourrait dire que s'il y a aujourd'hui une crise de la ville, une crise de l'urbanisme, c'est que plus aucun appareil n'arrive à s'imposer aux autres. D'où la fascination un peu rétro pour les montages, les collages urbains, les références historiques, etc.

\section{BIBLIOGRAPHIE}

Arendt Hannah, Qu'est-ce que la politique ?, Paris, Seuil, 1995.

Baldwin John W., Paris, 1200, Paris, Aubier, 2006

Benjamin Walter, Le Narrateur, Paris, Seuil, 1995.

Benjamin Walter, Fragments, Paris, PUF, 2001.

Bodei Remo, La Sensation de déjà vu, Paris, Seuil, 2007.

Damisch Hubert, L'origine de la perspective, Paris, Flammarion, 1999.

Déotte Jean-Louis, Qu'est-ce qu'un appareil ? Benjamin, Lyotard, Rancière, Paris, L'Harmattan, 2006.

Déotte Jean-Louis, « Le cinéma, un appareil de déviance », Appareil, nº 6, 2010.

Detienne Marcel, Les maîtres de vérité en Grèce archaïque, Paris, Maspéro, 1973.

Lyotard Jean-François, La condition postmoderne, Paris, Minuit, 1979.

Oehler Dolf, Le spleen contre l'oubli. Juin 1848. Baudelaire, Flaubert, Heine, Herzen, Paris, Payot, 1996.

Schefer Jean-Louis, L'Homme ordinaire du cinéma, Paris, Éd. de l'étoile, 1997.

Valero Vicente, Expérience et pauvreté. W. Benjamin à Ibiza (1932-1933), Rodez, Le Rouergue-

Chambon, 2003.

\section{NOTES}

1. Hannah Arendt, Qu'est-ce que la politique?, Paris, Seuil, 1995, p. 40.

2. Ibid., p. 42.

3. Marcel Detienne, Les maîtres de vérité en Grèce archaïque, Paris, Maspéro, 1973.

4. Cf. Les analyses par Hubert Damisch des Cités idéales d'Urbino, Berlin, Baltimore (L'origine de la perspective, Paris, Flammarion, 1999).

5. Walter Benjamin, Fragments, Paris, PUF, 2001.

6. Walter Benjamin, Le Narrateur, Paris, Seuil, 1995 ; voir plus loin. Jean-François Lyotard, La condition postmoderne, Paris, Minuit, 1979.

7. La question devient alors celle-ci : à partir de quand la multitude urbaine est-elle devenue une masse caractérisée par la fantasmagorie ? Il est évident que le partage se fait ici entre Berlin d'un côté (ce que confirmera a contrario le texte de Hegel) et Paris et Londres de l'autre. Le personnage d'Hofmann observe les passants du haut de son bow-window à travers une lunette de théâtre, le résultat c'est une série d'images du format d'une vignette. Tout autre sera le point de vue 
londonien de Dickens qui observe la foule à travers la vitrine d'un café, attendant de la rejoindre comme s'il était aimanté par ce flux continu : cinématographique?

8. Vicente Valero, Expérience et pauvreté. W. Benjamin à Ibiza (1932-1933), Rodez, Le RouergueChambon, 2003.

9. John W.Baldwin, Paris, 1200, Paris, Aubier, 2006.

10. Dolf Oehler, Le spleen contre l'oubli. Juin 1848. Baudelaire, Flaubert, Heine, Herzen, Paris, Payot, 1996.

11. Voir Jean-Louis Déotte, Qu'est-ce qu'un appareil? Benjamin, Lyotard, Rancière, Paris, L'Harmattan, 2006 et Remo Bodei, La Sensation de déjà vu, Paris, Seuil, 2007.

12. Jean-Louis Schefer, L'Homme ordinaire du cinéma, Paris, Éd. de l'étoile, 1997.

13. Le lien entre représentations et politique de la ville est esquissé dans un petit essai consacré au théâtre prolétarien pour enfants. Commentant le travail social et politique de son amie Asja Lacis dans les villes soviétiques où errent après la guerre civile des bandes d'enfants abandonnés, Benjamin montre que la mise en scène théâtrale par les enfants permet à la fois de lutter contre leur propension à la fantasmagorie et d'innerver la perception visuelle par l'action motrice. Cette mobilisation des énergies juvéniles grâce à la représentation doit libérer des signes qui sont autant de fragments de projets pour une action politique à venir (voir Jean-Louis Déotte, «Le cinéma, un appareil de déviance ", Appareil, $\mathrm{n}^{\circ}$ 6, 2010).

\section{AUTEUR}

\section{JEAN-LOUIS DÉOTTE}

Professeur des Universités (philosophie esthétique), département de philosophie Paris 8, coresponsable du laboratoire de philosophie (EA 1468), Responsable du programme « Arts, Appareils, Diffusion » de la MSH Paris Nord. 